\title{
Joint Segmentation and Tracking of Object Surfaces in Depth Movies along Human/Robot Manipulations
}

\author{
Babette Dellen, Farzad Husain and Carme Torras \\ Institut de Robòtica i Informàtica Industrial, CSIC-UPC, Llorens i Artigas 4-6, 08028 Barcelona, Spain \\ \{bdellen,shusain,torras\}@iri.upc.edu
}

Keywords: Range data, Segmentation, Motion, Shape, Surface fitting.

Abstract: A novel framework for joint segmentation and tracking in depth videos of object surfaces is presented. Initially, the 3D colored point cloud obtained using the Kinect camera is used to segment the scene into surface patches, defined by quadratic functions. The computed segments together with their functional descriptions are then used to partition the depth image of the subsequent frame in a consistent manner with respect to the precedent frame. This way, solutions established in previous frames can be reused which improves the efficiency of the algorithm and the coherency of the segmentations along the movie. The algorithm is tested for scenes showing human and robot manipulations of objects. We demonstrate that the method can successfully segment and track the human/robot arm and object surfaces along the manipulations. The performance is evaluated quantitatively by measuring the temporal coherency of the segmentations and the segmentation covering using ground truth. The method provides a visual front-end designed for robotic applications, and can potentially be used in the context of manipulation recognition, visual servoing, and robot-grasping tasks.

\section{INTRODUCTION}

During human or robotic manipulations, we face the challenge of having to interpret a large amount of visual data within a short period of time. The data from the sensors needs to be structured in a way that makes task-relevant visual information more accessible. The recognition of objects and scene context in a temporally consistent manner plays here a central role.

Moreover, in manipulation tasks, the use of 3D information is of particular importance, since accurate grasping and object manipulation require knowledge about both the 3D shape of the objects and their 3D context, e.g., to avoid collisions. For depth acquisition, stereo set-ups, laser-range scanners, or time-offlight depth sensors are commonly used. Recently, the release of the Kinect camera (Kinect, 2010), a depth sensor based on a structured light system, has opened new possibilities for acquiring depth information in real time.

A traditional way to process the visual data in manipulation tasks is to use geometric models for recognizing objects in the image and to track them using conventional tracking paradigms along the manipulation (Kragic, 2001). In this case, exact object models need to be defined prior to the task, which, considering the variability of an object's appearance in the image, has the drawback that the system may not easily adapt to new scenarios.

In this work, we approach the problem from a different angle. Our main contribution and aim is the creation of consistent segmentations of depth images, into geometric surfaces, along a depth video and the tracking of segments along the movie. Starting from a known initial segmentation of the first frame into surface segments, we show in this paper how this information can be exploited in a consecutive frame to group the current depth values into segments. This way, information from the previous frame can be efficiently recycled, and segment labels can be kept throughout the sequence, enabling tracking of surface patches.

The robot can use such a representation to draw conclusions about scene content (Aksoy et al., 2011), to guide its own movements (visual servoing), or to use surface information for the planning of grasping movements (Taylor and Kleeman, 2002), or even in a learning-by-demonstration context (Agostini et al., 2011; Rozo et al., 2011). At a later stage, higher-level information about objects may enter the task by describing objects through their composite 3D surfaces (Hofman and Jarvis, 2000).

The paper is structured as follows: In Section II, we discuss related work. The proposed algorithm is 
introduced in Sections III-IV. Then, in Section V, the results for different human/robot manipulations are presented. Future work is sketched in Section VI.

\section{RELATED WORK}

Joint segmentation and tracking has previously been performed mostly for color image sequences (Abramov et al., 2010; Deng and Manjunath, 2001; Patras et al., 2001; Wang, 1998; Wang et al., 2009; Grundmann et al., 2010). In a recent work, the color images were segmented by finding the equilibrium states of a Potts model (Abramov et al., 2010). Consistency of segmentations obtained along the movie and the tracking of segments were achieved through label transfer from one frame to the next using optic flow information. This way, the equilibrium states in the current frame could be encountered more rapidly. The resulting segments represent regions of uniform color and usually do not coincide with the object surfaces in a geometric sense, which we would desire for our system. The solutions found by Abramov et al. (2010) cannot be easily adapted to our problem, because color segmentation and depth segmentation are inherently different problems. Surfaces cannot be defined based on local properties only, which increases the difficulty of the problem considerably.

Other methods for video segmentation are usually performing independent segmentations of each frame and then try to match segments (Deng and Manjunath, 2001; Patras et al., 2001; Wang, 1998; Grundmann et al., 2010). This is problematic because segmentations have to be computed from scratch for every frame, which has consequences on both the computational efficiency of the method and the temporal consistency of the results. For cluttered scenes, the partition of the segmentation tends to change from one frame to the next, and temporal coherence of the segmentations is prone to be impaired because of this effect.

In another work, segmentation and multi-object tracking were performed simultaneously using graphical models (Wang et al., 2009). Observed and hidden variables of interest describing the appearance and the states of objects are jointly considered and used to formulate the objective as a Markov random field energy minimization problem. Different from our method, depth measurements do not enter the framework, and objects are defined based on their 2D appearance alone. Also, objects of interest are defined in the first frame and are then tracked along the sequence. While the method delivers convincing results, energy minimization is computationally expen- sive and efficient optimizations would have to be developed to make the approach more practical.

To the authors' knowledge, little work has been done in this field using depth information as the primary vision cue for segmentation and tracking. Parvizi and $\mathrm{Wu}$ performed multiple object tracking using an adaptive depth segmentation method (Parvizi and $\mathrm{Wu}, 2008$ ). Time-of-flight depth was used to segment each frame independently by finding the connected components based on an absolute depth distance measure. The segments of adjacent frames were then associated with each other using a depth histogram distribution. However, this depth segmentation method is rather simple and does not partition the data into distinct surfaces. As a consequence, boundaries defined by changes in 3D shape (curvature) cannot be detected, which constitutes a major difference in comparison to our method. In addition, each movie frame is segmented from scratch. In the case of surface segmentation, this can be rather costly. Furthermore, the temporal consistency of the segmentations will degrade with increasing clutter in the scene.

In Lopez-Mendez et al. (2011), upper body tracking of a human using a range sensor (Microsoft Kinect) is performed. Their technique is limited to the human beings only as they use a prior model of human body.

\section{OVERVIEW OF THE METHOD}

Our method for depth-video segmentation consists of three parts: (i) Segment transfer and seeding, (ii) re-estimation of surface models and grouping, and (iii) a consistency check and respective re-grouping of pixels (see Fig. 1).

In the first part (i), labels of the depth image obtained at frame $F^{t}$ are transferred to the next frame $F^{t+1}$. Surface models that have been fitted to the depth of frame $F^{t}$ for all segments are transferred as well. A seed is created for each label by comparing the predicted depth with the measured depth in the projected segment area. If the distance of the measured depth and model depth is smaller than a threshold, the respective pixel is accepted and used as a seed for constructing the full segment region in the current frame.

In part (ii), the surface models are re-estimated for each segment using the current depth values of the respective seed. Non-seed points are grouped in connected components, and then assigned to the closest surface in the neighborhood. Connectedness of found segments is evaluated, and the labeling is adjusted accordingly. 


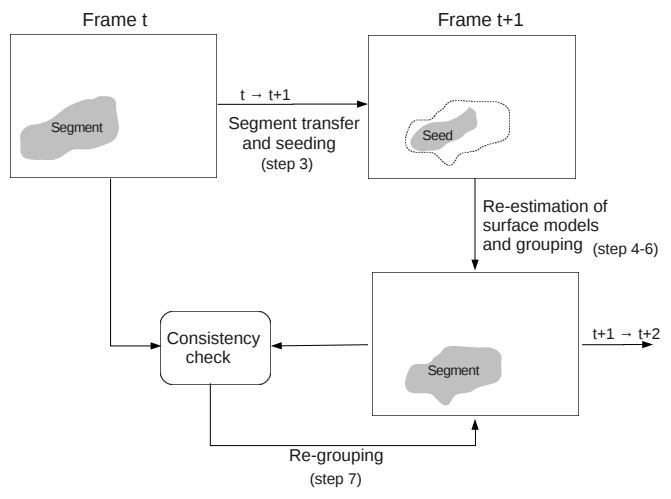

Figure 1: Schematic of the method. Segment regions obtained for frame $F^{t}$ are transferred to frame $F^{t+1}$. The points lying inside a given segment region are compared with the respective surface model, and only those points which fit the surface model are marked as seed points of the given segment in frame $F^{t+1}$. Then, the surface parameters of the respective segment model are re-calculated using the depth values of the seed. Using these models, the depth of points outside the seed region can be predicted for each segment, and the remaining points are assigned to the closest segment surface, taking some proximity constraints into account. Finally, the obtained segmentation for frame $F^{t+1}$ is compared with the previous segmentation for frame $F^{t}$. Only if an inconsistency is detected, the affected segments are re-grouped using region growing and shrinking until the problem is resolved.

Finally, in part (iii), the temporal consistency of segments along the video is checked. Because of the high frame rate of the Kinect, it can be assumed that changes between frames (at least in the given scenarios) are small, implying that a segment cannot grow or shrink out of proportion from one frame to the next. In case that such a temporal consistency problem is detected, the points of the affected segments are regrouped until the problem is resolved, using a clearly defined termination criterion.

A Microsoft Kinect sensor along with the Kinect package of ROS (Robot Operating System) is used to acquire sequences of depth images $F^{1}, \ldots, F^{t}, F^{t+1}, \ldots F^{t+n}$ for different scenarios. The algorithm is implemented in Matlab. Each frame contains the color values $(r, g, b)$ and $(x, y, z)$ values from the depth sensor, resulting in a matrix of size $m \times n \times 6$, where $m$ and $n$ are the spatial dimensions of the image grid. However, only the $(x, y, z)$ values are used by the proposed algorithm.

\section{ALGORITHM}

Our algorithm for joint segmentation and tracking consists of the following consecutive steps (Fig. 1):
1. Initial labeling: A labeling $l^{t}(u, v)$ of the initial frame at $t=1$ is computed using an algorithm proposed in Dellen et al. (2011). Here $u$ and $v$ are the indexes of the image grid. Color segments are extracted from the color image using a standard algorithm (Felzenszwalb and Huttenlocher, 2004) for different resolutions. Quadratic surfaces are fitted to the color segments using depth data, and the best patches are selected from the hierarchy of resolutions, creating a new segmentation. This segmentation is further improved by merging those patches that are considered to describe the same surface. This is achieved by a recent graph-based clustering method for surfaces based on Kruskal's algorithm (Kruskal, 1956). This gives a segmentation of the image into $k$ disjoint segments $s_{1}, \ldots, s_{j}, \ldots, s_{k}$ with $s_{i} \cap s_{j}=\varnothing$ and respective labels $1, \ldots, j, \ldots, k$.

2. Model fitting: A quadratic surface model $f_{j}^{t}(x, y)$ of the form

$$
z=a x^{2}+b y^{2}+c x+d y+e,
$$

with surface parameters $a, b, c, d$, and $e$ is fitted to each segment $s_{j}$ by performing a LevenbergMarquardt minimization of the mean square distance

$$
E_{j}=1 / n_{j} \sum_{(u, v) \in s_{j}}\left[z_{e}(u, v)-z(u, v)\right]^{2}
$$

of the measured depth points $z(u, v)$ from the estimated model depth $z_{e}(u, v)=f[x(u, v), y(u, v)]$. Here, $n_{j}$ is the number of measured depth points in the area of segment $s_{j}$. The chosen model type allows modeling of planar and curved surfaces, e.g., cylinders and spheres. The iterative solver (Levenberg-Marquardt minimization) enables us to use the solution obtained for the previous time step as the starting location. This leads to temporal consistency. For the initial frame we set the starting location as zero. In our case, the algorithm converged in an average of 4 iterations.

3. Seeding: In order to update the segmentation grid according to the current frame, the first step should be to unlabel the points $(u, v)$ that do not fit the surface. We achieve this by generating seeds. For each point $(u, v)$ of frame $F^{t+1}$, we find the projected label $p=l^{t}(u, v)$ from the previous segmentation and define a seed labeling for $F^{t+1}$ according to

$m^{t+1}(u, v)= \begin{cases}p & \text { if }\left|z_{e}(u, v)-z(u, v)\right|<\tau_{p}, \\ 0 & \text { otherwise }\end{cases}$ 
with $z_{e}(u, v)=f_{p}^{t}[x(u, v), y(u, v)]$, and

$$
\tau_{p}=\sum_{(u, v) \in s_{p}^{t+1}}\left|z_{e}(u, v)-z\right| /\left(\rho n_{p}\right),
$$

where $s_{p}^{t+1}$ is the segment $s_{p}^{t}$ projected into the current frame $F^{t+1}, n_{p}$ is the number of pixels in the area of $s_{p}$, and $\rho$ is a constant. This defines a labeling $l^{t+1}(u, v)=p$ for all $(u, v) \in s_{p}^{t+1}$.

4. Updating models: Now the surface model parameters need to be updated, so that they can model the current state of the surfaces. For each label $j$ we obtain a surface model $f_{j}^{t+1}(x, y)$ by applying the fitting procedure of step (2) to the seed of $s_{j}$, consisting of all the points $(u, v)$ for which $m^{t+1}(u, v)=j$ holds.

5. Grouping of non-seed points: Once we have updated the model parameters, we can determine the new labels of non-seed points. All points $(u, v)$ with $m^{t+1}(u, v)=0$ are grouped into connected components. For each connected component $c_{i}$, we search the neighborhood of all boundary points $(u, v)$ within a radius $r_{1}$ for seed points. If a seed point is found, its label is added to the list of potential labels $L_{i}=\left\{l_{1}, l_{2}, ..\right\}$ for $c_{i}$. For each label $q \in L_{i}$, we compute the distance

$$
d_{q}(u, v)=\left|f_{q}^{t+1}[x(u, v), y(u, v)]-z(u, v)\right| .
$$

For all $(u, v) \in c_{i}$, we can set

$$
l^{t+1}(u, v)=\arg \left[\min \left(\left\{d_{l_{1}}(u, v), d_{l_{2}}(u, v), . .\right\}\right)\right],
$$

defining the labeling for the non-seed points.

6. Ensuring connectedness of the current labeling $l^{t+1}(u, v)$ : The assignment of new labels does not guarantee that the segments defined by the new labeling represent connected components. The segments should only get disconnected if the surface becomes occluded by another surface(s). For example in Fig. 2(b) it can be observed that at some point in time the background was disconnected. In order to avoid false non-connected segments, we unlabel them if their size is less than the minimum allowable segment size (we use 800 pixels, but this could be changed adaptively depending on the scenario) and assign them the label of the segment with which the largest boundary is shared.

The current labeling $l^{t+1}(u, v)$ is updated accordingly. Since connectedness is ensured, $l^{t+1}(u, v)$ represents a segmentation of frame $F^{t+1}$ into $k$ segments.

7. Regrouping to maintain temporal consistency: Since Kinect camera can deliver a high frame rate of up to $30 \mathrm{fps}$, we can reasonably assume relatively small motion of objects between consecutive frames. This implies that a segment cannot grow or shrink in size out of proportions from one frame to the next. For each segment $s_{j}$ of frame $F^{t+1}$, we compute the segment size ratio $\triangle a_{j}=a_{j}^{t+1} / a_{j}^{t}$, where $a_{j}^{t+1}$ and $a_{j}^{t}$ are the size of $s_{j}$ in frame $F^{t}$ and $F^{t+1}$, respectively. If $\triangle a_{j}>1+\delta$ or $\triangle a_{j}<1-\delta$, a label assignment error is assumed. In this case, we compute the relative change for all direct segment neighbors of $s_{j}$. If relative change is almost equal (no. of pixels added in one segment $s_{j} \approx$ no. of pixels removed in the other $s_{i}$ ), we extract the contact line between the two segments and assign all points $(u, v)$ within a radius $r_{2}$ of the contact line to $s_{i}$ until the ratio $\triangle a_{j} \approx 1$, providing the termination criterion.

8. Steps $2-8$ are repeated for the next frame using $l^{t+1}(u, v)$ as initial labeling.

\section{RESULTS}

\subsection{Segmentation Results}

We tested the algorithm for several depth movies showing human and robot manipulations of objects. Videos are provided as supplementary material at http://www.iri.upc.edu/people/bdellen/Movies.html.

As an example of a typical manipulation action, we show segmentation results for a human hand grasping a carton box and placing it on top of a cylindrically shaped paper roll (see Fig. 2). In Fig. 2(a), selected calibrated depth images acquired with the Kinect are shown. In Fig. 2(b), the segmentation results obtained by our method are shown. Fig. 2(c) shows a ground truth segmentation of surfaces as perceived by a human for comparison. Fig. 2(d) shows results obtained using video-segmentation algorithm based on color for comparison (Grundmann et al., 2010). The segments are color-coded, where each color corresponds to a unique segment label. With our method not all surfaces could be completely recovered in the initial segmentation, due to the limited depth resolution. However, in the following, a change of position of the carton box allows correctly 


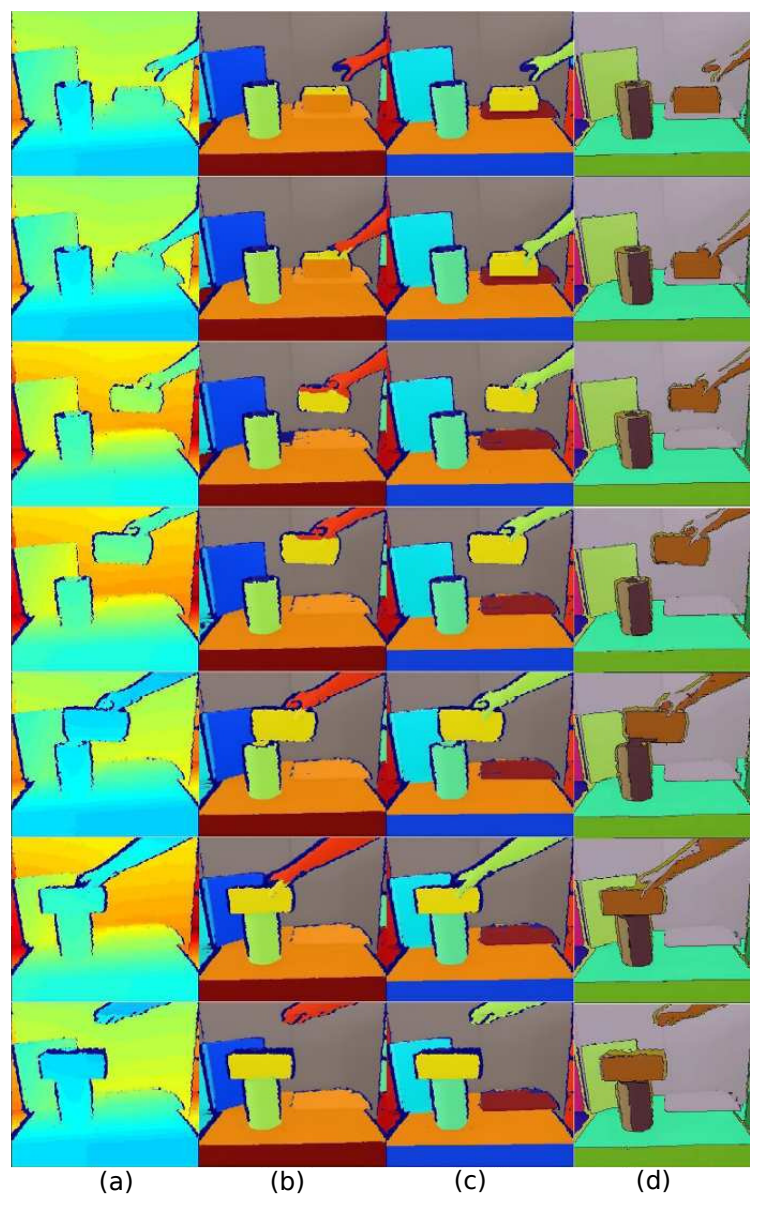

Figure 2: Hand grasping a carton box. (a) Depth image (Kinect). (b) Video segmentation results using our method. (c) Human segmentation used as ground-truth (d) Color image video segmentation using (Grundmann et al., 2010).

segmenting and tracking the surfaces (see Fig. 2(b)). In comparison with the ground truth (Fig. 2(c)), it can be seen that a small percent of false label assignments happens during the manipulation of the carton box, since local depth information becomes insufficient. The problem is resolved to a certain degree by regrouping (see step (7) of the algorithm). In comparison, in the color-based approach (shown in Fig. 2(d)), the hand is merged with both the background and the carton box. This is an inherent problem in algorithms which rely on color information alone, because different surfaces cannot be guaranteed to always have a different color.

Next, we present results for a human hand rolling a green ball forward and then backwards with its fingers (see Fig. 3). The ball and the hand are correctly segmented and tracked along the image sequence, even though the hand is changing its shape during the

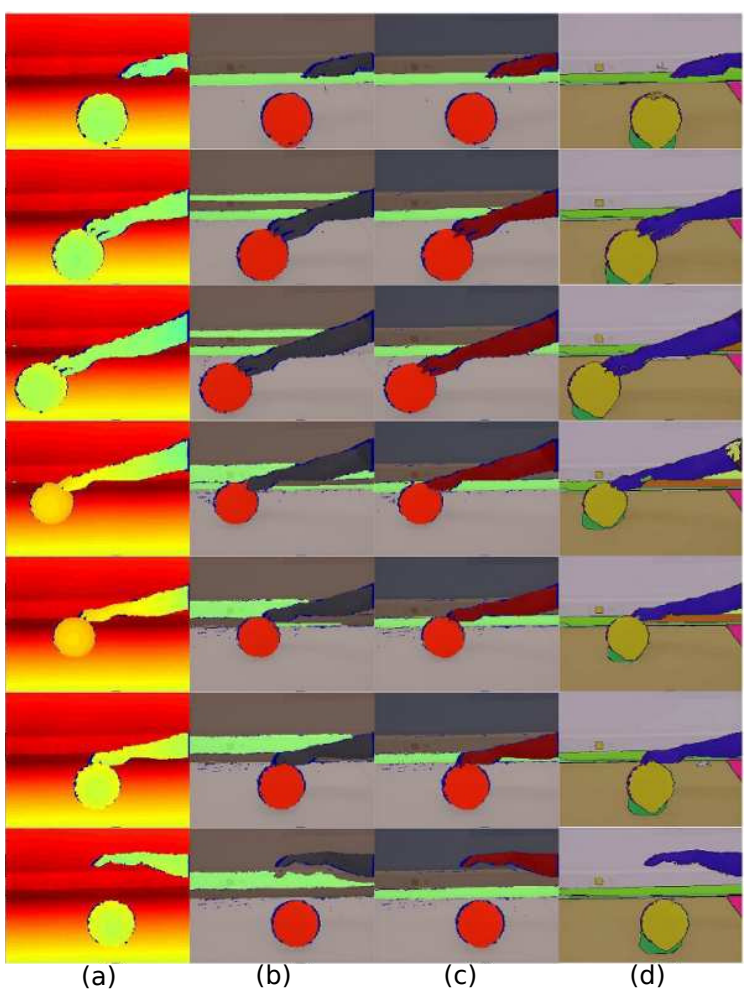

Figure 3: Hand rolling a ball. (a) Depth image (Kinect). (b) Video segmentation results using our method. (c) Human segmentation used as ground-truth (d) Color video segmentation using (Grundmann et al., 2010).

motion (see Fig. 3 (b)). Ground-truth segmentations and the results of the color-based video segmentation proposed by Grundmann et al. (2010) are shown for comparison in Fig. 3(c-d), respectively.

We further show segmentation results for a movie where a robot arm grasps a cylindrically-shaped paper roll and moves it to a new position (see Fig. 4). During the movement, objects in the background become occluded. Nevertheless, the sequence is correctly segmented and both the robot arm and the paper roll are tracked along the movie, as can be seen in Fig. 4(b). In the color-based video segmentation (see Fig. 4(d)), the robot arm gets over segmented and the carton box, which is lying on the table, gets merged with the table (undersegmented).

Finally, we demonstrate a scenario in which multiple segments are tracked simultaneously. Fig. 5 shows selected frames of a plant movie. It can be seen that as the plant is being displaced, multiple segments are tracked jointly through the scene. Notice that two leaves in the initial segmentation were under segmented so they are tracked in the same way in upcoming frames. 


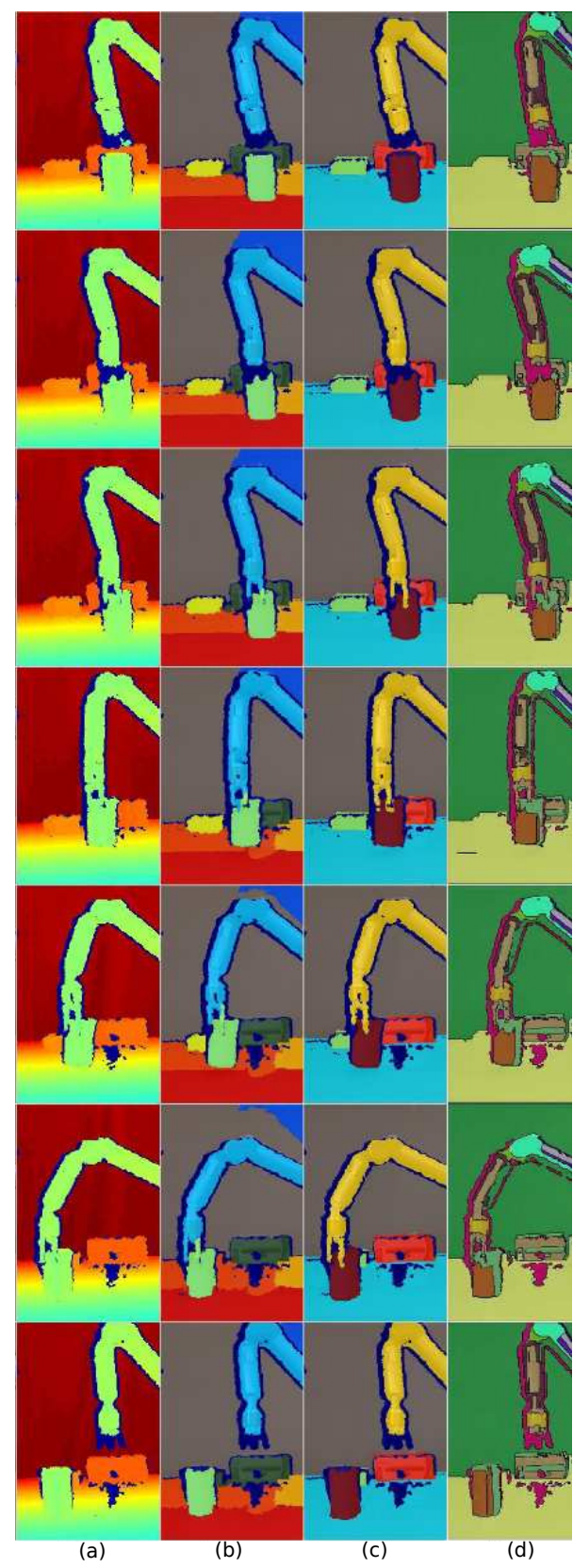

Figure 4: WAM robotic arm grasping and displacing a paper roll. (a) Depth image (Kinect). (b) Video segmentation results using our method. (c) Human segmentation used as ground-truth (d) Color video segmentation using (Grundmann et al., 2010).

\subsection{Quantitative Evaluation}

We use the segmentation covering metric described in Arbelaez et al. (2009) to determine how closely the segmentation results match the ground truth segmentation. Human annotated color images are used

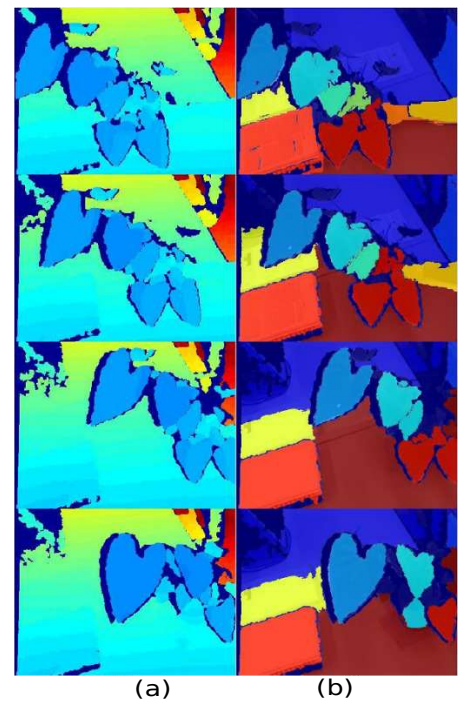

Figure 5: Plant being displaced. (a) Depth image (Kinect). (b) Video segmentation results using our method.

as ground truth (column (c) of Fig. 2, 3, 4). For one frame, the segmentation covering metric is defined as

$$
C\left(S^{\prime} \rightarrow S\right)=\frac{1}{N} \sum_{R \in S}|R| \cdot \max _{R^{\prime} \in S^{\prime}} O\left(R, R^{\prime}\right)
$$

where $N$ is the total number of pixels in the image, $|R|$ the number of pixels in the region $R$, and $O\left(R, R^{\prime}\right)$ is the overlap between the regions $R$ and $R^{\prime}$ defined as

$$
O\left(R, R^{\prime}\right)=\frac{\left|R \cap R^{\prime}\right|}{\left|R \cup R^{\prime}\right|}
$$

Fig. 6 (blue line) shows a plot of the segmentation covering metric for the segmentation results of our algorithm, corresponding to videos of Fig. 2, 3, 4. The segmentation covering metric is computed for frames taken at fixed time intervals. The segmentation covering metric of the segmentation result shown in Fig. 6(c,blue line) has a lower value compared to the other examples because in this case the background got over-segmented initially and the algorithm tries to track these over-segmented surfaces in upcoming frames, as can be seen in Fig. 4(b). We also plotted the segmentation covering metric for the color-video-segmentation results obtained with a graph-based video segmentation algorithm (Grundmann et al., 2010) in red for comparison. For the given set of movies, our method clearly outperforms the method described in (Grundmann et al., 2010). For the plant movie (Fig. 5) similar results were obtained. The segmentation covering metric gave a value of 0.89 and 0.83 for the initial and the final frame, respectively, when compared to a human perceived segmentation. 

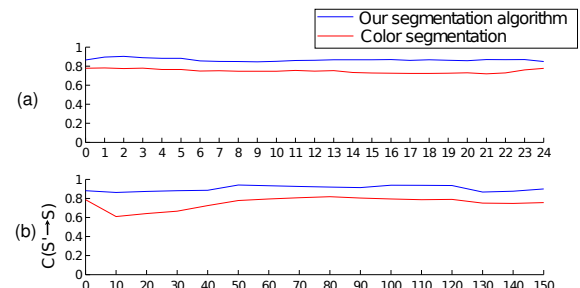

(c)

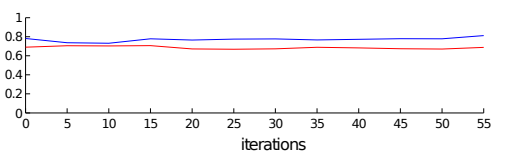

Figure 6: Segmentation covering metric for the results obtained with our segmentation algorithm (in blue) and with the graph-based method (Grundmann et al., 2010) (in red) for the scenes shown in (a) Fig. 2, (b) Fig. 3 and (c) Fig. 4.
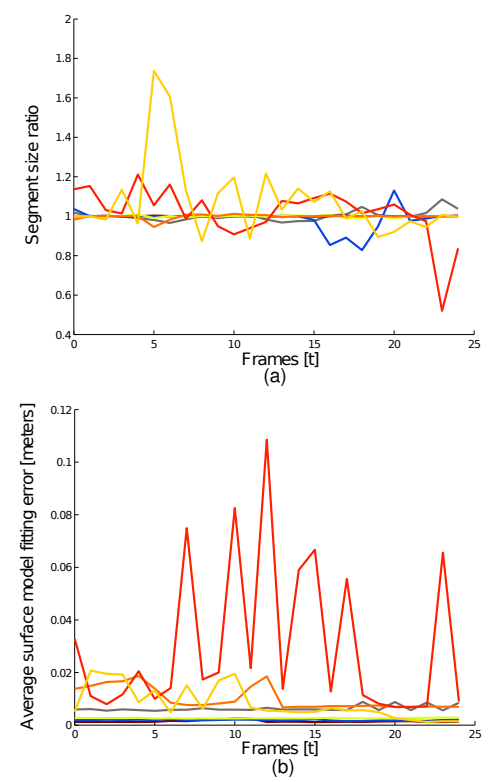

Figure 7: Evaluation of segmentation coherence and surface fitting error. (a) Segment size ratio $\triangle a_{i}$ as a function of the frame number for all segments $s_{i}$ shown in Fig. 2. (b) Surface fitting errors $E_{i}$ as a function of the frame number. Line colors correspond to the segment-label colors of Fig. 2(c).

We evaluated the coherence of the segmentations by measuring the segment size ratio $\triangle a_{i}$ of the segments $s_{i}$ as a function of time. For the example of Fig. 2, results are shown in Fig. 7(a). The line-plot colors correspond to the label colors used in Fig. 2(b). The segment size ratios are fluctuating between 0.8 and 1.2, indicating that temporal consistency is maintained.

We further compared the depth predicted by the surface models of the segments with the measured (ground truth) depth and computed the fitting error for each segment (see Fig. 7(b)). Except for the hand, the fitting error remains below 0.02 meters. The larger errors measured for the hand are caused by small segmentation errors at its large boundary lines due to its fast motion, which affects the tracking procedure negatively.

\subsection{Parameter Choices}

The algorithm contains two important parameters, i.e., $\rho$ and $\delta$, which may require tuning. The remaining parameters $r_{1}$ and $r_{2}$ are less critical.

For our set-up, the parameter $\rho$ required for the segmentation of consecutive frames was determined only once and not altered during the different experiments, except for one experiment, for which it needed to be increased. With our chosen value of $\rho=1.7$, an average of $74 \%$ of points per segment served as seed points.

By evaluating the segment-size ratio over time (see Fig. 7(a)), which, in case of successful tracking, stayed in between 0.8 and 1.2, we set parameter $\delta$ equal to 0.2 , providing a reasonable bound on the segment-size change of $20 \%$. This parameter, once set, was not varied throughout the experiments. The remaining parameters were set to $r_{1}=10$ pixels and $r_{2}=15$ pixels

\section{CONCLUSIONS}

We presented a novel algorithm for joint segmentation and tracking of object surfaces, defined by their geometric shapes. Segments obtained for the first frame are used to initialize the segmentation procedure of the next frame, and so on. The main novelties of the proposed method are (i) the use quadratic surface models for seeding and in the context of a tracking problem (steps 2-3), (ii) a labeling technique for non-seed points, defined by Eq. 6 (see step 6 of the algorithm), and a re-grouping strategy enforcing temporal consistency between frames (step 7). We tested the algorithm for several movies acquired with the Kinect showing human and robot manipulations of objects. The algorithm allowed us to segment and track the main object surfaces in the scene, despite frequently occurring occlusions, limited resolution of the depth images, and shape changes of the hand and the robot gripper. However, some problems still remain. Occasionally, depth differences between surfaces are too small, resulting in assignment conflicts that cannot be resolved by the method as it is. In the future, we aim to incorporate additional mechanisms for improving 
the robustness of the method in this respect. Furthermore, we are currently developing mechanisms for generating new segments in addition to the ones that have been determined in the first frame, which will be important in case new objects are entering the scene. This will also allow segmenting images from scratch in the future, i.e., the initial frame. The segment consistency check and the following re-grouping procedure are currently conducted using hard thresholds, which we plan to make adaptive in the future. We plan to make our tracking algorithm more robust to occlusions and noise by using shape information from all the previous time steps. A way to achieve this would be building dynamic shape models (Cremers, 2006).

We provided a quantitative evaluation of the method using human-annotated ground truth. Obtaining ground-truth for video is however a very tedious procedure and thus poses us limits. Since there is no implementation of a similar algorithm performing joint segmentation and tracking in depth space available, we compared our method to a standard colorvideo segmentation algorithm (Grundmann et al., 2010). We could show that our method outperformed color-video segmentation for the videos analyzed. However, this comparison may not be entirely fair, since we are using a different feature, i.e., depth, and not color.

Currently, the method needs $\sim 1.92$ seconds to process one frame of size $430 \times 282$ pixels in Matlab on Intel $3.3 \mathrm{GHz}$ processor. With an efficient $\mathrm{C} / \mathrm{C}++$ implementation of the method, we expect to gain realtime performance, which is one of our next goals.

\section{ACKNOWLEDGEMENTS}

This research is partially funded by the EU projects GARNICS (FP7-247947) and IntellAct (FP7-269959), and the Grup consolidat 2009 SGR155. B. Dellen acknowledges support from the Spanish Ministry of Science and Innovation through a Ramon y Cajal program.

\section{REFERENCES}

Abramov, A., Aksoy, E. E., Dörr, J., Wörgötter, F., Pauwels, K., and Dellen, B. (2010). 3d semantic representation of actions from efficient stereo-image-sequence segmentation on gpus. In 5th Intl. Symp. 3D Data Processing, Visualization and Transmission.

Agostini, A., Torras, C., and Wörgötter, F. (2011). Integrating task planning and interactive learning for robots to work in human environments. In IJCAI, Barcelona, pages 2386-2391.
Aksoy, E. E., Abramov, A., Dörr, J., Ning, K., Dellen, B and Wörgötter, F. (2011). Learning the semantics of object-action relations by observation. Int. J. Rob. Res., 30(10):1229-1249.

Arbelaez, P., Maire, M., Fowlkes, C., and Malik, J. (2009). From contours to regions: An empirical evaluation. In CVPR, pages $2294-2301$.

Cremers, D. (2006). Dynamical statistical shape priors for level set-based tracking. IEEE TPAMI, 28(8):1262 1273.

Dellen, B., Alenya, G., Foix, S., and Torras, C. (2011). Segmenting color images into surface patches by exploiting sparse depth data. In IEEE Workshop on Applications of Computer Vision, pages 591 -598.

Deng, Y. and Manjunath, B. (2001). Unsupervised segmentation of color-texture regions in images and video. IEEE TPAMI, 23(8):800 -810.

Felzenszwalb, P. F. and Huttenlocher, D. P. (2004). Efficient graph-based image segmentation. Intl. J. of Computer Vision, 59(2):167-181

Grundmann, M., Kwatra, V., Han, M., and Essa, I. (2010). Efficient hierarchical graph-based video segmentation. In CVPR, pages $2141-2148$.

Hofman, I. and Jarvis, R. (2000). Object recognition via attributed graph matching. In Proc. Australian Conf. on Robotics and Automation, Melbourne, Australia.

Kinect (2010). Kinect for xbox 360. In http://www.xbox.com/en-US/kinect.

Kragic, D. (2001). Visual Servoing for Manipulation: Robustness and Integration Issues. $\mathrm{PhD}$ thesis, Computational Vision and Active Perception Laboratory, Royal Institute of Technology, Stockholm, Sweden.

Kruskal, J. B. (1956). On the Shortest Spanning Subtree of a Graph and the Traveling Salesman Problem. In Proc. of the American Mathematical Society.

Lopez-Mendez, A., Alcoverro, M., Pardas, M., and Casas, J. (2011). Real-time upper body tracking with online initialization using a range sensor. In IEEE Intl. Conf. on Computer Vision Workshops, pages 391 -398.

Parvizi, E. and Wu, Q. (2008). Multiple object tracking based on adaptive depth segmentation. In Canadian Conf. on Computer and Robot Vision, pages 273-277.

Patras, I., Hendriks, E., and Lagendijk, R. (2001). Video segmentation by map labeling of watershed segments. IEEE TPAMI, 23(3):326 -332.

Rozo, L., Jimenez, P., and Torras, C. (2011). Robot learning from demonstration of force-based tasks with multiple solution trajectories. In 15th Intl. Conf. on Advanced Robotics, pages $124-129$.

Taylor, G. and Kleeman, L. (2002). Grasping unknown objects with a humanoid robot. In Proc. of Australasian Conf. on Robotics and Automation, pages 191-196.

Wang, C., de La Gorce, M., and Paragios, N. (2009). Segmentation, ordering and multi-object tracking using graphical models. In IEEE 12th Intl. Conf. on Computer Vision, pages $747-754$.

Wang, D. (1998). Unsupervised video segmentation based on watersheds and temporal tracking. IEEE Transactions on Circuits and Systems for Video Technology, 8(5):539-546. 